\section{Understanding decoding}

During protein synthesis, the ribosome accurately selects aminoacyl tRNAs in accordance with mRNA codons in the decoding center in the A site. The tRNA selection process proceeds in two consecutive steps: initial selection and proofreading. During proofreading, the

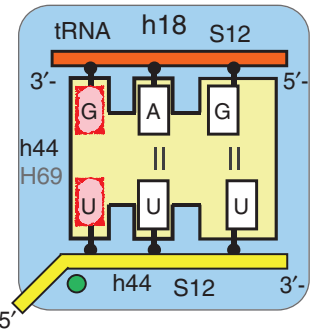
ribosome has a second chance to reject the tRNA if it does not match the codon in the A site. Although the mechanism underlying the decoding process was previously studied on the basis of structures of the isolated $30 \mathrm{~S}$ subunit, Yusupova and colleagues have now determined six X-ray structures of the bacterial $70 \mathrm{~S}$ ribosome, in which they modeled cognate and near-cognate states of the decoding center at the proofreading step by using long mRNA and tRNAs whose anticodons carried a mismatch corresponding to the first, second or third position of the mRNA triplet in the A site. They show that the $30 \mathrm{~S}$ subunit undergoes an identical domain closure upon binding of either cognate or near-cognate tRNA. Remarkably, single $\mathrm{U} \bullet \mathrm{G}$ and $\mathrm{G} \bullet \mathrm{U}$ mismatches in the first or second codon-anticodon position, which were expected to form standard wobble base pairs, instead adopt Watson-Crick-like base pairs, owing to positional restrictions imposed by the decoding center on these base pairs. By contrast, no such restraints exist for the third base pair. The authors propose that geometrical restriction of the first two base pairs of the codon-anticodon duplex by the very tight decoding pocket causes the near-cognate tRNA to be expelled from the ribosome. (Nature doi:10.1038/nature10913, published online 21 March 2012)

AH

\section{Un-arrested development}

Auxins are plant hormones that regulate a broad range of biological processes, including embryogenesis, stem elongation, leaf formation and fruit development. These tryptophan-like small molecules elicit their physiological responses by binding to the F-box-containing transport inhibitor response 1 (TIR1) family of proteins and causing the degradation of the auxin/indole-3-acetic acid (Aux/IAA) family of transcriptional repressors. The exact mechanisms by which auxins regulate so many different processes in plants is not clear, and studying this system is challenging because there are six TIR1/Auxin-signaling F-box (TIR1/AFB) proteins and 29 known Aux/IAA proteins in Arabidopsis thaliana. Estelle and colleagues used several techniques, including in vitro auxin-binding assays, yeast two-hybrid and plant growth assays, to show that the TIR1/AFB proteins and the Aux/IAA proteins act as co-receptors for auxin, with dramatically different binding affinities and dose-response relationships, depending on which TIR1/AFB-Aux/IAA pair was studied. It seems likely that some TIR1/AFB-auxin-Aux/IAA complexes form at a very low intracellular auxin concentration in vivo, whereas other complexes do not assemble until the intracellular auxin concentration exceeds a specific value. This suggests that different TIR1/AFB-Aux/IAA pairs may regulate different physiological processes in plants. Additional research is needed to determine which of the 174 possible TIR1/ AFB-Aux/IAA pairs actually form in vivo and to elucidate how the formation of a specific TIR1/AFB-auxin-Aux/IAA ternary complex leads to a specific physiological event. (Nat. Chem. Biol. doi:10.1038/ nchembio.926, published online 1 April 2012)

$J M F$

Written by Joshua M. Finkelstein, Arianne Heinrichs, Steve Mason \& Michelle Montoya

\section{Small RNA-mediated DNA repair}

The repair of DNA double-strand breaks (DSBs) is a complex, tightly regulated process. Small noncoding RNAs, such as miRNAs and siRNAs, play many diverse regulatory roles, but their functions in DSB repair have remained largely unexplored. Qi and colleagues have now examined the repair of Arabidopsis thaliana DSBs and report that small RNAs of $\sim 21$ nucleotides in length, derived from both sense and antisense sequences flanking DSBs, are upregulated following DNA damage. Termed DSB-induced small RNAs (diRNAs), their production depends on the kinase ATR, which promotes DNA repair in response to single-stranded DNA, and RNA polymerase IV, which transcribes siRNAs in plants. RNA-dependent RNA polymerases that produce double-stranded RNA (dsRNA) from single-stranded RNA are also necessary for diRNA production, as are Dicer-like proteins, which cleave dsRNAs into shorter fragments. Argonaute 2 (AGO2), a component of the RNA-induced silencing complex, binds mature diRNAs, and loss of AGO2 significantly reduces diRNA levels and impedes DSB repair. However, diRNA expression does not affect RNA-directed DNA methylation, histone $\mathrm{H} 2 \mathrm{AX}$ phosphorylation, or the levels of key DNA repair proteins, so the mechanism for their effects on DNA repair is still unclear. Importantly, diRNAs were also detected in human cells, where DSB repair depends on Dicer and AGO2. This suggests that diRNAs have an evolutionarily conserved role in DNA repair, and the authors propose their function may be to recruit specific repair factors. (Cell 149, 101-112, 2012)

$S M$

\section{Mad1 attachment}

The spindle checkpoint protects the cell from chromosome missegregation and is activated when unattached kinetochores are detected during mitosis. The checkpoint proteins are recruited to the unattached kinetochores and inhibit the anaphase-promoting complex until the kinetochores attain proper spindle attachment. The Mad1-Mad2 complex is key to the spindle checkpoint, yet how targeting to kinetochores is achieved is not well understood. Yu, Luo and colleagues now examine Mad1 targeting, determining the structure of the human Mad1 C-terminal domain (CTD). The CTD is dimeric, forming a coiled-coil tail with a globular head, and it bears unexpected structural similarity to kinetochore-targeting components of the $\mathrm{Ndc} 80$ complex. This similarity led the authors to investigate CTD's role in Mad1 targeting. In human cells, truncation of the CTD resulted in a decrease in Mad1 targeting to kinetochores, suggesting that the CTD domain is an important determinant for Mad1 targeting during mitosis but not its only one. Additional mutagenesis revealed that a conserved Arg-Leu-Lys (RLK) motif within the CTD was important for targeting. Previous work in yeast showed that this motif was needed for checkpoint-stimulated binding of Mad1 by the spindle checkpoint proteins Bub1-Bub3, and cells with RLK motif mutations were deficient in the spindle checkpoint. The authors here found that in human cells, depletion of Bub1 reduced Mad1 localization at kinetochores, similar to what they observed with RLK mutants. The findings suggest that Bub1 acts as a receptor for Mad1 CTD but that contacts with additional kinetochore proteins play a role in Mad1 targeting. Future studies will undoubtedly tease out other Mad1 interaction partners as well as the upstream signals that lead to its recruitment to the kinetochore. (Proc. Natl. Acad. Sci. doi:10.1073/ pnas.1118210109, published online 9 April 2012) MM 